\title{
Tattoos: ancient body art may assist in medical emergencies
}

\author{
Sarah Chadwick • Mamta Shah
}

Received: 30 January 2013 / Accepted: 5 February 2013 / Published online: 15 February 2013

(C) Springer-Verlag Berlin Heidelberg 2013

\begin{abstract}
Tattooing, like medicine, is an ancient art form. However, in the UK, tattooing of minors is illegal except when performed for medical reasons. We present a 15 -yearold type I diabetic, who being prone to hypoglycaemic attacks, had a permanent medical alert tattoo on his forearm, with his parents' consent, whilst on holiday abroad. Tattooing to convey a medical message is employed by many adults for reasons as diverse as anaphylaxis to do not resuscitate orders. We present the patient and propose that clinicians may wish to consider supporting tattooing to convey a medical alert in young people, particularly those at risk of life-threatening complications, such as hypoglycaemia.
\end{abstract}

Keywords Tattoo $\cdot$ Diabetes

\section{Case report}

Failure to recognise hypoglycaemia in diabetic patients can be life-threatening and if mistaken for drug or alcohol intoxication, it can leave the individual in danger [1]. To avoid this, parents of a 15-year-old boy with diabetes in our department consented for him to have his diagnosis permanently tattooed onto his forearm whilst on holiday abroad, as tattooing of minors is currently illegal in the UK (Fig. 1).

Tattooing, needle insertion of permanent ink or dyes into the dermis of the skin, is safe for diabetics as long as it is done in a reputable establishment and the wound is cared for [2]. Diabetics undergoing tattooing should ensure good

M. Shah

Department of Burns and Plastic Surgery,

Royal Manchester Children's Hospital, Hathersage Road,

Manchester M13 9PL, UK

S. Chadwick $(\square)$

University of Manchester, 3.614 Stopford Building,

Oxford Road, Manchester M13 9PT, UK

e-mail: slchadwick@doctors.org.uk

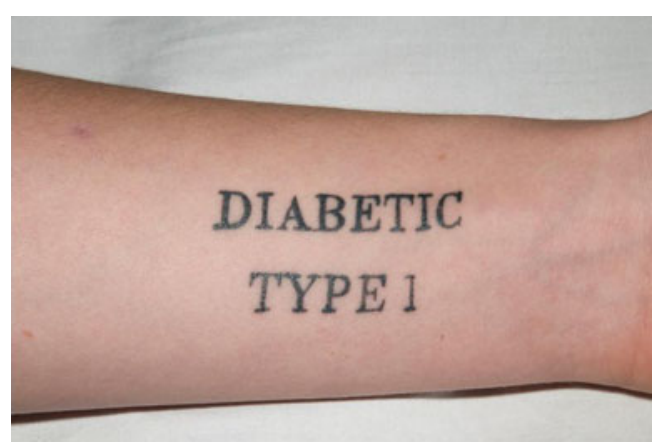

Fig. 1 Our patient's tattoo, on the volar surface of his forearm, clearly shows his diagnosis as a type I diabetic. The site is easily accessible in an emergency

glycaemic control and avoid areas at risk of microvascular/neuropathic disease and common insulin injection sites.

Tattooing, like medicine, is an ancient art form which could offer a potentially life-saving solution for young people affected by life-threatening medical conditions. However, it is currently illegal in the UK except "when the tattoo is performed for medical reasons" [3]. We propose that doctors should consider supporting young people requesting tattoos on medical grounds, and for maximum effectiveness, the medical fraternity should promote a recognised symbol and site for alert tattoos to allow rapid recognition and prompt treatment of emergencies.

\section{References}

1. DiabetesHealth (2008) Mr Universe assaulted by police during low blood sugar episode. [Online] Available at: http://www.diabetes health.com/read/2008/12/17/5081/mr-universe-assaulted-by-policeduring-low-blood-sugar-episode/. Accessed 25 Jan 2013

2. Sperry K (1991) Tattoos and tattooing. Part I: history and methodology. Am J Forensic Med Pathol 12:313-319

3. Tattooing of Minors Act (1969) (c.24), London: HMSO 